light food by the mouth. Later she was given bi-palatinoids of carbonate of potash and iron, both of which are indicated in these cases. In certain districts of the Rhön mountains and the Bavarian Alps, in which the inhabitants are mostly vegetarians, gastric ulcer is very uncommon, and vegetarian diet gives to the system a large amount of potassium salts. I think that in this patient the healed cicatrix of an old ulcer had partly broken down and caused a renewal of symptoms ; I know of very many cases with a similar history. I therefore venture to suggest that perhaps in many cases of so-called simple irritative dyspepsia such a lesion is present though the ulcer may have hitherto been latent. There is another point to mention-viz, the absence of free hydrochloric acid in the vomited matters-and this is generally observed in cancer of the stomach, but lately it has been shown to occur in about one-third of the cases of ulcer, and so its diagnostic significance is but slight.

Broadhurst-gardens, N.W.

\section{RAPID SUPERVENTION OF SYMPTOMS OF} POISONING FOLLOWING THE APPLICATION OF A BELLADONNA \section{PLASTER.}

BP WILliaM J. HOWARTH, M.D. VICT.,

LATE HIOUSE SURGEON AT THE ROYAL INFIRMARY, MANCHESTER, AND LOUGHBOROUGH INFIRMARY AND DISPENSARY.

THE following notes of a case of poisoning by belladonna appear to be worthy of record on account of the extreme rapidity with which the symptoms followed the application of a belladonna plaster to the lumbar region.

A man aged fifty-seven years, on Des. 13th, 1893, after a powerful stroke with a pickaxe while at work as a gardener, experienced a sharp stabbing pain in the loins. He had some difficulty in moving after it, but friction with mustard oil gave him some relief. He suggested the application of a belladonna plaster, and one about six inches by four inches in size was procured and applied over the surface, which had been rendered hypermemic by the stimulating oil. He then proceeded to walk home, a distance of about two miles and a half. He could only walk slowly, and in about three-quarters of an hour he noticed that his mouth was becoming dry and his tongue dry and swollen. The skin under the plaster felt very cold, and there was a peculiar numbness in his hands and feet. This increased so much in intensity that he hardly knew when his feet touched the ground, and at the same time the power of moving his legs became more feeble ; consequently, he had much difficulty in "guiding his steps." He began to feel "dizzy," and noticed that the lights in the shop windows seemed to flicker. Everything he looked at was streaked with lines, alternating black and white in colour, and each appeared to be about a quarter of an inch in thickness. Later his sight began to grow dim and his mind became very confused. He arrived home in about an hour and a quarter, and after having vomited he became quite unconscious, having no recollection of anything which took place until 4 A. is. I saw him about an hour later, and found his pupils widely dilated and not reacting to light. His breathing was somewhat hurried and deep. The lips were apparently very dry and the tongue dry and coated. There was delirium, incoherent and muttering, and of an amused type; he frequently burst out laughing in quite a hearty manner. The pulse was 70 per minute (usually it is about 55). The tendon reflexes were very pronounced. Muscular power was very feeble; he was quite unable to change his position. The facial muscles and fingers frequently twitched. Having been informed that he had on a belladonna plaster it was removed, and the plaster still acihering to the skin was cleaned with olive oil and afterwards well washed. There was no abrasion of the skin under the plaster, but it was markedly hyperamic from the previous vigorous treatment. I then gave him half a grain of the extract of physostigma about 5.30 P.M., and repeated it twice at intervals of two hours. About 3 A.M. (twelve hours after the application of the plaster) he commenced to perspire very freely, and discharged a copious amount of urine-none having been passed previously to this-when all the symptoms rapidly improved, so that by 12 noon on the next day he seemed to be almost well again, complaining only of weakness in the legs. The dryness of the mouth and the swollen condition of the tongue had quite disappeared, the reflexes were not nearly so pro. nounced, and the pulse-rate was about 60 per minute. The pupil remained dilated and sluggish, and only assumed its normal condition after three days.

Pendlebury, Manchester.

\section{climital hotes:}

\section{MEDICAL, SURGICAL, OBSTETRICAL, AND} THERAPEUTICAL.

\section{ON THE VALUE OF COUGHING-TAXIS IN THE REDUCTION OF HERIA.}

By George WherRy, M.B. CAMB , F.R.C.S. ENG., \&c , UNIVERSITY LECTURER ON SURGERY, CAMBRIDGE.

THAT cough which so often produces a hernial protrusion may be used with the greatest advantage during reduction. The abdominal parts concerned are in the most favour. able position for reduction of hernia while the patient is coughing; the taxis should then be applied by careful fingers after the following method. On beginning the attempt at reduction the surgeon places himself and his patient in the most comfortable and favourable position for gentle continuous manipulations; the patient should be recumbent, with the buttocks rather raised and the thigh flexed on the abdomen, while the surgeon would usually prefer to sit by the bedside. During the whole of the time that taxis is used the patient must cough, only stopping for rest or retching; the operator's efforts should be continuous even during these pauses, and his gentle but firmly applied pressure will be aided by every cough of the patient; also during retching I have thought some advantage has been gained. In this method the sensation to the surgeon is that the hernia is reduced rather by the coughing of the patient than by the working of the fingers of the manipulator. I have during several years past so often succeeded in reducing hernix of ail varieties that I can strongly advise this plan, feeling sure that it only requires to be known and practised to be appreciated. The last case which occurred to me I was called to see at the hospital at two o'clock in the morning, "very cross and dogged," as the spiritual Diarist records. I found a middle-aged woman with a femoral bernia with urgent signs of strangulation. She had been under the care of two medictl men, who had, with taxis, used chloroform. The patient coughed during ten or fifteen minutes while my quite gentle but continuous taxis was used; the hernia was first gradually reduced in bulk, and the last little button returned with a jump into the abdominal cavity. A good recorery followed.

The finger placed in the healtby abdominal ring while coughing is practised by the person under examination will enable the operator to realise that the alternating contractions and relaxations of the boundaries of the ring are most favourable for the return of a hernia if pressure be skilfully practised. In commending this plan I have only my own experience to go upon, but it is now sufficiently large to enable me to express myself with confidence in its favour. Coughing-taxis as well as tussio-taxis would be a name as good as any other hyphened hybrid for this manœurre, which 1 hope may prove as useful in other hands as it has done in mine. Cambridge.

\section{ACUTE ABSCESS OF THE LEFT FRONTAL SINCS} SIMULATING ORBITAL CELLULITIS.

By M. P. MAyo Collier, M.S. LoNd., F.R C.S. ENG, VICE-PRESIDENT OF THE BRITISH LARYNGOLOGICAL ASSOCIATION ETC.

A YouTH nineteen years of age came to the North-West London Hospital in August last complaining of severe pain and swelling in the left orbit. He had suffered from tro previous attacks, but neither of them had been so severe as the present one. The patient was suffering intense pain in the frontal region, and his temperature, as taken in the ontpatients" room, was $103^{\circ} \mathrm{F}$. On a closer examination of the 